\title{
Cost effective robust rule calibration system
}

\author{
P. Greeff* and O. Kruger \\ National Metrology Institute of South Africa (NMISA), Pretoria, South Africa
}

Received: 25 June 2014 / Accepted: 12 July 2014

\begin{abstract}
One of the main calibration services of African NMIs (National Metrology Institutes) is the measurement of tapes and rules. This is mainly regulated by legal metrology and OIML (International Organisation of Legal Metrology) specifications are therefore referenced. Specifically, OIML R-35 is the standard to which rules or line scales must conform. The accuracy of most African NMIs systems however, cannot prove conformance to this specification. This article will detail the development of a new, cost effective, line scale calibration system, which will have accuracy better than the specification prescribed. The system was locally developed and its design is based on off-the-shelf components and open source software. It is also ready-for-upgrade to an absolute system. The system and details of the line detection algorithm will be presented.
\end{abstract}

Keywords: Rule calibration, line detection

\section{Introduction}

The need for more efficient traceable rule and tape calibrations for some local laboratories and most African NMIs has become apparent. This statement will be considered in terms of current requirements and methods. A novel system which was specifically designed to address this need will be shown. Details around this system will be discussed and verification results presented. Finally the results are used to prove that it is feasible that the new system can resolve the identified need.

One of the main calibration services of African NMIs are the measurements of tapes and rules, which are performed with a comparative measurements technique. Two rules or line scales are placed next to each other: one will be standard (calibrated traceable to the metre, typically by a laser interferometry system) and the other the UUT (Unit Under Test). The adjacent lines of the UUT and standard are compared and the UUT deviation estimated after aligning the two scales (see Fig. 1). Calibration is done by eye, usually with a magnification glass. This method results in a traceable accuracy of not better than $\pm 0.5 \mathrm{~mm}$. OIML specification [1] is referenced for the accuracy requirements since the calibration of rules in Africa is typically driven by legal metrology. For example OIML class 1 requires a $\pm 0.5 \mathrm{~mm}$ accuracy for a one metre rule.

A measuring accuracy of $\pm 0.5 \mathrm{~mm}$ however cannot prove conformance to a specification requiring $\pm 0.5 \mathrm{~mm}$ (see Fig. 2).

The measurement method mentioned above can however be improved, since it relies heavily on the metrol-

\footnotetext{
* Correspondence: pgreeff@nmisa.org
}

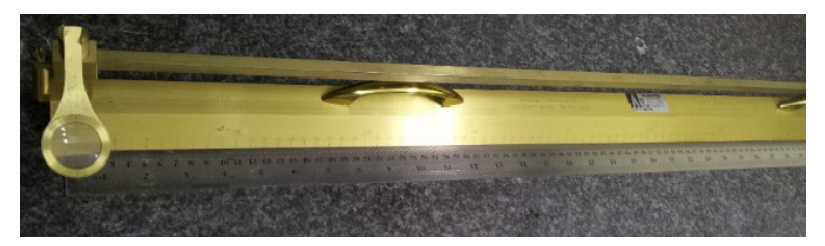

Fig. 1. Photo of a steel rule being calibrated against a typical standard rule.

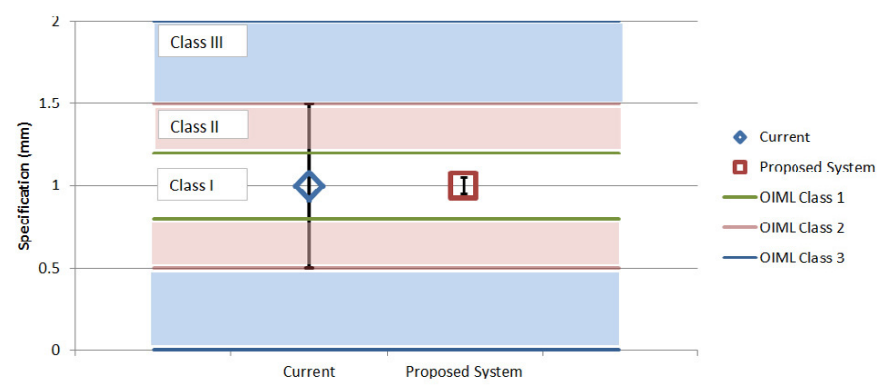

Fig. 2. Current and proposed system uncertainties versus OIML class requirements.

ogist ability to guess the line centre at a fraction of a $1 \mathrm{~mm}$ repeatedly. Furthermore, if more than one metrologist measures the same rule, the reproducibility can be significantly affected due to human error.

The main motivation of this project therefore is to upgrade the current method using automated line centre detection software.

Secondly, the technology to detect line centre and similar image attributes can be used in the future for new systems or even high accuracy optical standard calibrations. 


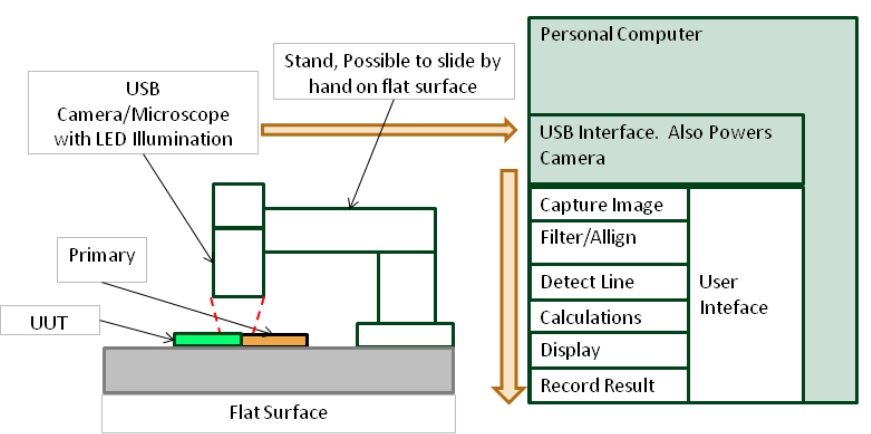

Fig. 3. Main system component overview.

\section{Development requirements}

Based on the discussion above, requirements for the new system was determined as follows:

1. Reduce the human error factor in line centre estimation by implementing a camera and an algorithm. This must be:

a. Robust (find line centre and rule edges repeatability, for different surfaces).

b. Easy to use.

c. Able to determine the UUT deviation from the calibrated standard rule.

2. Improve the accuracy of the comparison method from $\pm 0.5 \mathrm{~mm}$ to $\pm 0.05 \mathrm{~mm}$.

3. System must be cost effective.

4. Easy to maintain, modify or upgrade (For example: upgrade to an absolute, instead of a comparison, type system).

It was decided to develop the system with open source software. This will make it possible for any laboratory to give inputs and to join in the technical development of line scale metrology in Africa.

\section{System overview}

The system utilizes 'off-the-shelf' components and inhouse developed open source application (see Fig. 3) to calibrate rules, line scales or tapes, by comparison (placing two rules next to each other).

A USB microscope camera is used to capture an image. This is processed in real time by the software, which then determines key features. The software can currently detect the line centre and edge. This result is used to (1) calibrate the image frame $y$-axis based on a known distance or (2) determine the distance between two lines on two different line scales (comparison method). It can also be used to position the camera above the line centre (absolute method).

The application was develop in Python which is free and open [2]. The OpenCV package [3] was used along with Numerical Python (NumPy) [4] for capturing the image from the microscope, manipulating the image and applying the algorithm. A graphical user interface was developed using PyQt [5].

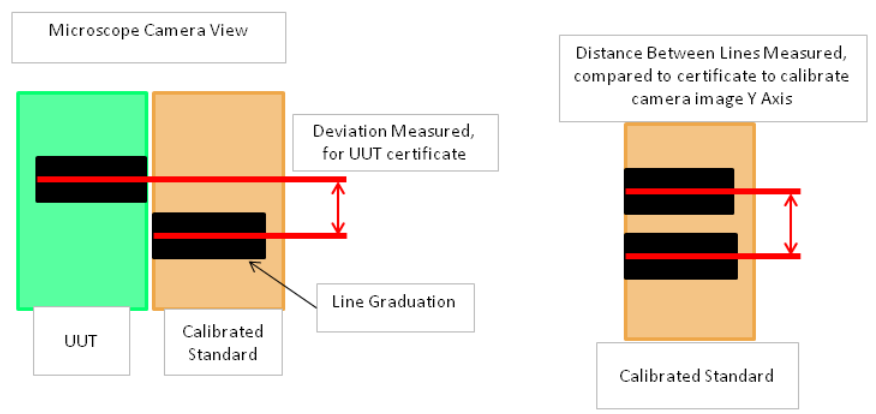

Fig. 4. The comparison principal.

The main concept of the system is the calibration of the image $Y$-axis by determining the line centre distance of a previously calibrated standard rule and using this calibration factor to measure the distance between the current UUT and standard line (see Fig. 4). Within this lie a few important assumptions, for example:

(1) Both scales zero marks are aligned perfectly relative to each other.

(2) The scale to image size ratio does not change, i.e. the camera height, zoom and image zoom rectangle sizes stay the same.

(3) The UUT and standard rule are at the exact same temperature and at $20{ }^{\circ} \mathrm{C}$.

(4) The UUT and standard rule scale axes are parallel to each other and to the camera movement axis.

(5) The UUT and standard rule scale are in the same plane.

(6) The camera image sensor is parallel, or the camera itself is perfectly orthogonal, to the scale plane.

(7) The camera does not distort the image non-linearly.

(8) The scale lines are horizontal on the image.

Some of these assumptions can be corrected for (for example (1 and 3$)$ ), but the rest will contribute to the measurement uncertainty, which will be discussed later.

Figure 5 visualizes the step by step process developed to make the calibration seamless. The first step is to load the calibration certificate of the standard from a text file and to enter relevant measurement data, such as the CTE (coefficient of thermal expansion) and which segments to be used for the camera calibration. The next step is to setup the calibration itself. Firstly the camera, then the line scale type options and finally the user can run various tests to check if the detection is working sufficiently. If it is not, various advanced settings can be used to optimize line detection.

The second calibration process step is to calibrate the microscope camera image, specifically the area enlarged. This determines a linear factor for the $Y$-axis of the zoomed image, based on the calibration certificate values.

Lastly the comparison can be commenced. The microscope is positioned above the required lines and the measurements are made at the user's command. The results can be viewed and is also saved in text file, as selected by the user. 




Fig. 5. User interface process flow.

\subsection{Algorithm detail}

Various options were considered for the centre detection algorithm. Based on the requirements it was decided that the algorithm must be robust and easy to understand and therefore easy to adjust certain parameters.

An average value for each row (it assumed that the lines on the image is perfectly horizontal) is calculated and compared to trigger level. If the row average level is above the trigger level it can be either a line start or end, depending on the previous state. Finally if it is a valid line the line centre is calculated, either using a weighted mean or the middle point (of the start and end line). This line centre is then added to the line centre list, which can then be used by the rest of the application to calibrate the image $y$-axis or determine the distance between a target line and current line. Figure 6 shows the main steps described above.

Image pre-processing includes using a ROI (Region of Interest) instead of an array copy, to speed up the process (though not that critical). Various options are selectable or adjustable, for example blur amount, image rotation, thresh holding method (if active), averaging and level filtering.

Another technique which is common for line detection is the Hough Transform [6]. It is feasible to implement such an algorithm since it is already method in OpenCV. This method can however be considered in future work.

\subsection{CTE correction}

If the UUT and standard does not have the same CTE (Coefficient of Thermal Expansion), then it is required to apply a correction, especially if the temperature differs significantly with respect to the reference temperature of $20^{\circ} \mathrm{C}$.

For example if the standard is made from Brass (CTE of $\left.18 \mathrm{ppm} /{ }^{\circ} \mathrm{C}\right)$ and the UUT of steel $\left(11.5 \mathrm{ppm} /{ }^{\circ} \mathrm{C}\right)$ it will result in a $\mathrm{CTE}$ difference of $6.5 \mathrm{ppm} /{ }^{\circ} \mathrm{C}$. This will



Fig. 6. Detection control algorithm steps.

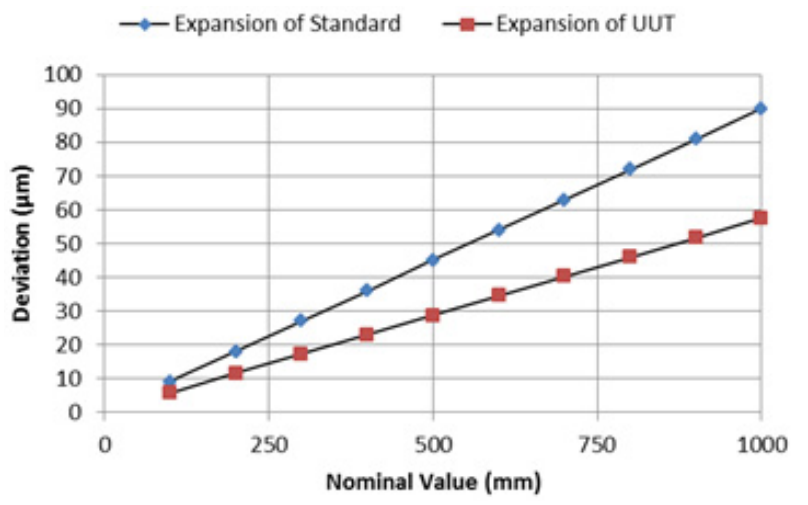

Fig. 7. Difference in thermal expansion for a brass and steel rule at $25{ }^{\circ} \mathrm{C}$.

cause a difference of expansion of $32.5 \mu \mathrm{m}$ over $1 \mathrm{~m}$ for $5{ }^{\circ} \mathrm{C}$. Figure 7 illustrates this difference.

The following equation was derived to adjust the raw comparison data, based on CTE's and current temperature:

$L_{U U T}=\left[L_{\text {meas }}+L_{S T D}\left(1-\alpha_{S T D} \Delta T\right)\right]\left(1+\alpha_{U U T} \Delta T\right)$.

Derived from the standard linear equation for thermal expansion:

$$
L_{\text {Corrected }}=L_{\text {Actual }}(1+\alpha \Delta T)
$$

where $L_{U U T}$ is the corrected (referenced back to $20^{\circ} \mathrm{C}$ ) length of the UUT, $L_{\text {meas }}$ the comparison measurement result at the measured temperature, $L_{S T D}$ the calibration certificate length (at $20^{\circ} \mathrm{C}$ ), $\alpha_{S T D}$ and $\alpha_{S T D}$ the CTE of the UUT and the standard rule. Finally $\Delta T$ is the difference between the reference temperature and the measured temperature $\left(20^{\circ} \mathrm{C}-T_{\text {measured }}\right)$.

\subsection{Camera calibration method}

The comparison method requires that the image $Y$-axis is calibrated. Based on selected calibration certificate values, 'steps' are calculated. The step values are compared to the lines (distance between centres), and a linear factor calculated. Camera corrections are not applied since this a one dimensional measurement, and non-linearity is assumed small, especially as only an area (zoom rectangle) 


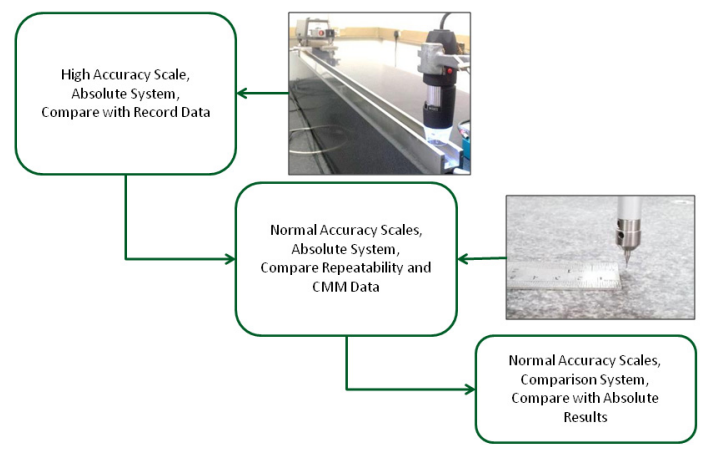

Fig. 8. System verification steps.

of the image is used for the comparison and not the whole image.

\section{Experiments and results}

Several experiments were conducted to test whether the algorithm and system works, see Figure 8. The experimental results were also used in the estimation of measurement uncertainty. Firstly, a high precision scale was measured using an absolute method (against a laser interferometer), but with the same camera and software as the comparison system.

Steel rules were measured with the absolute system and with a CMM next and compared, utilising the comparator system. These three steps are then combined to provide evidence for the system measurement ability.

\subsection{High accuracy scale absolute measurements}

The first experiment was the calibration of a standard which had sufficient historical data. This is used to test if the camera and line detection system works and whether it can be used to calibrate line scales, which in turn will be used for the testing of the comparator setup.

A high precision line scale was used, with records dating back to 1971. The USB microscope camera was mounted to a linear slide mounted with a laser interferometer for displacement measurements, while the scale is supported on a flat granite surface. The temperature of the scale is measured at both ends with a high accuracy digital thermometer. The setup results in large Abbe offset, which can be improved by changing the laser path setup. However the finally accuracy target for the comparison system is only $50 \mu \mathrm{m}$.

Table 1 contains the results for this calibration as compared to the average values (as calibrated at the NPL in 1985, BIPM in 1971 and 1980, all with an uncertainty less than $1 \mu \mathrm{m}$ ) for each $100 \mathrm{~mm}$ segment from 0 to $1000 \mathrm{~mm}$. The table shows that the maximum deviation from previous calibration is below $6 \mu \mathrm{m}$.

This falls within the uncertainty as mentioned above and proves that (1) the linear slide, laser interferometer and camera are measuring with sufficient accuracy and (2)

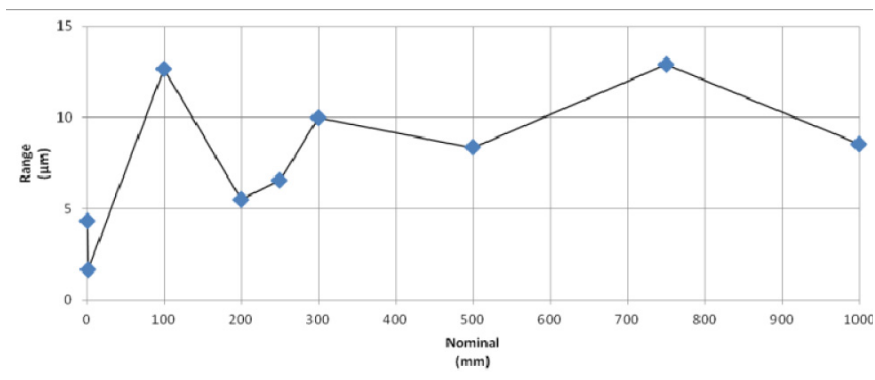

Fig. 9. Range over all measurements for each nominal point, for brass rule No. 7 .

Table 1. High accuracy scale test results.

\begin{tabular}{ccccr}
\hline & & Maximum $(\mu \mathrm{m})$ & -2.2 & 1.6 \\
\cline { 3 - 5 } $\begin{array}{c}\text { Nominal } \\
(\mathrm{mm})\end{array}$ & $\begin{array}{c}\text { average } \\
(\mathrm{mm})\end{array}$ & $\begin{array}{c}\text { Measurement } \\
\text { average } \\
(\mathrm{mm})\end{array}$ & $\begin{array}{c}\text { Deviation } \\
(\mu \mathrm{m})\end{array}$ & $\begin{array}{c}\text { Segment } \\
\text { deviation } \\
(\mu \mathrm{m})\end{array}$ \\
\cline { 3 - 5 } & 100.003 & 100.000 & -2.2 & \\
\hline 100 & 199.999 & 199.996 & -3.1 & -0.9 \\
200 & 299.999 & 299.995 & -3.9 & -0.7 \\
300 & 399.999 & 399.995 & -4.3 & -0.5 \\
400 & 499.999 & 499.995 & -3.7 & 0.6 \\
500 & 599.999 & 599.993 & -5.5 & -1.7 \\
600 & 700.000 & 699.996 & -3.8 & 1.6 \\
700 & 799.999 & 799.995 & -4.2 & -0.4 \\
800 & 899.998 & 899.994 & -3.6 & 0.6 \\
900 & 999.998 & 999.996 & -2.4 & 1.2 \\
\hline 1000 & & &
\end{tabular}

that the system can therefore be used to calibrated rules, which will be used to test the comparator system, if the effect on the line mark and edge does not influence the system significantly.

The $100 \mathrm{~mm}$ segment deviation of the measurements was also calculated. This is the step distance between each $100 \mathrm{~mm}$ line mark from zero. The average deviation decreases here to below $1 \mu \mathrm{m}$. This indicates that the system probably has an systematic error of about $4 \mu \mathrm{m}$, but measurement repeatability was also found to be similar (maximum range over each point was $3.8 \mu \mathrm{m}$ ). The scale was also supported on a flat surface and not the Bessel designated points, which could explain the maximum deviation at the scale centre.

\subsection{Reproducibility results}

The same rules were measured more than once, with different detections settings, to estimate the reproducibility of the absolute method.

Figure 9 shows the range over these measurements, per nominal point. The maximum standard deviation over these measurements (per nominal point) is $5 \mu \mathrm{m}$. 


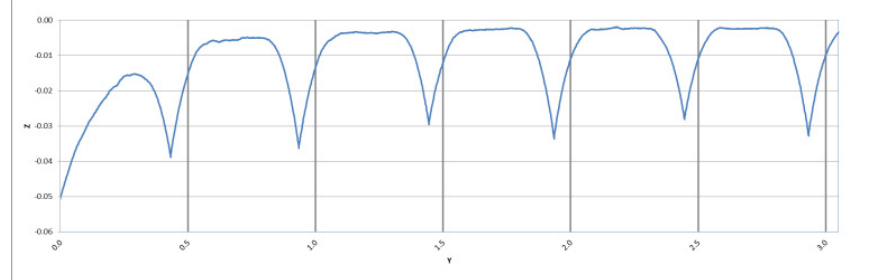

Fig. 10. CMM measurement results for edge type rule.

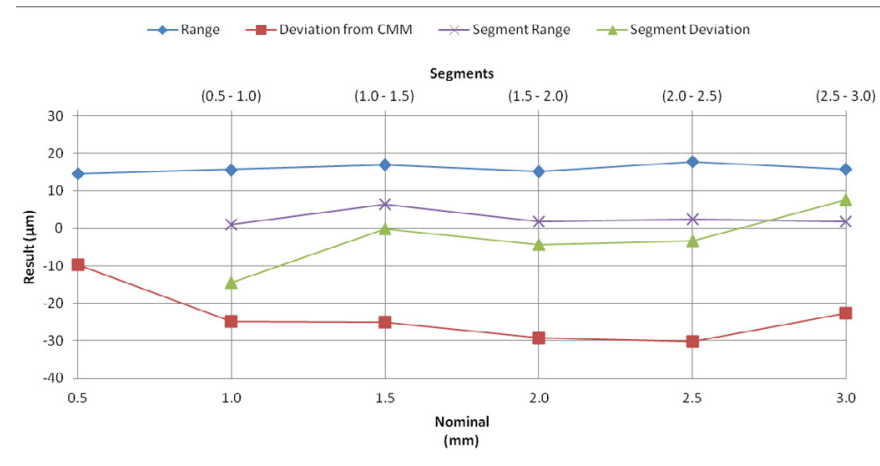

Fig. 11. Comparison between CMM and absolute system results.

\subsection{CMM comparison and edge measurements}

These results are sufficient evidence to move to the next step, which is considering the effect of line type difference between the high precision and general calibration rulers. This was investigated by measuring an engraved, $1 \mathrm{~m}$ steel rule, which has an edge or end surface for a zero mark with a high accuracy CMM.

The effect of the line scale mark and zero edges was investigated to confirm whether the results of the line scale marks of high accuracy scale can be used as verification of less accurate scales.

Figure 10 displays the results obtained with the CMM (Zeiss Prismo Ultra) measurement of the steel rule in $Y Z$ plot, with dimensions in $\mathrm{mm}$. A $0.5 \mathrm{~mm}$ probe was used for the calibration, with a CTE (Coefficient of Thermal Expansion) of of $11.5 \mathrm{ppm} /{ }^{\circ} \mathrm{C}$.

The CMM specification are $\pm\left(0.55+L \times 10^{-6}\right) \mu \mathrm{m}$, where $L$ is the measured distance in metre. This results in an basic estimation of $\pm 0.56 \mu \mathrm{m}$ for the CMM calibration.

Figure 11 compares the absolute line scale calibration system and the CMM results over the first 6 lines of the rule. Measurements with the absolute system were made with a standard deviation of $9 \mu \mathrm{m}$. The average of these values was used to determine the deviation from the CMM measurements. This gives a maximum deviation of $-30 \mu \mathrm{m}$.

If the line segments (e.g. from 0.5 to $1 ; 1$ to 1.5 ; etc.; removing the edge effect) are considered however, the maximum deviation is reduced to $15 \mu \mathrm{m}$.

Figure 12 displays two images from the software on a steel ruler, focused on the rule edge, which is used for the rule zero mark. The first image is the microscope image and the other the result of the zoom rectangle. The latter shows a 'grey' area (between the orange and red line)

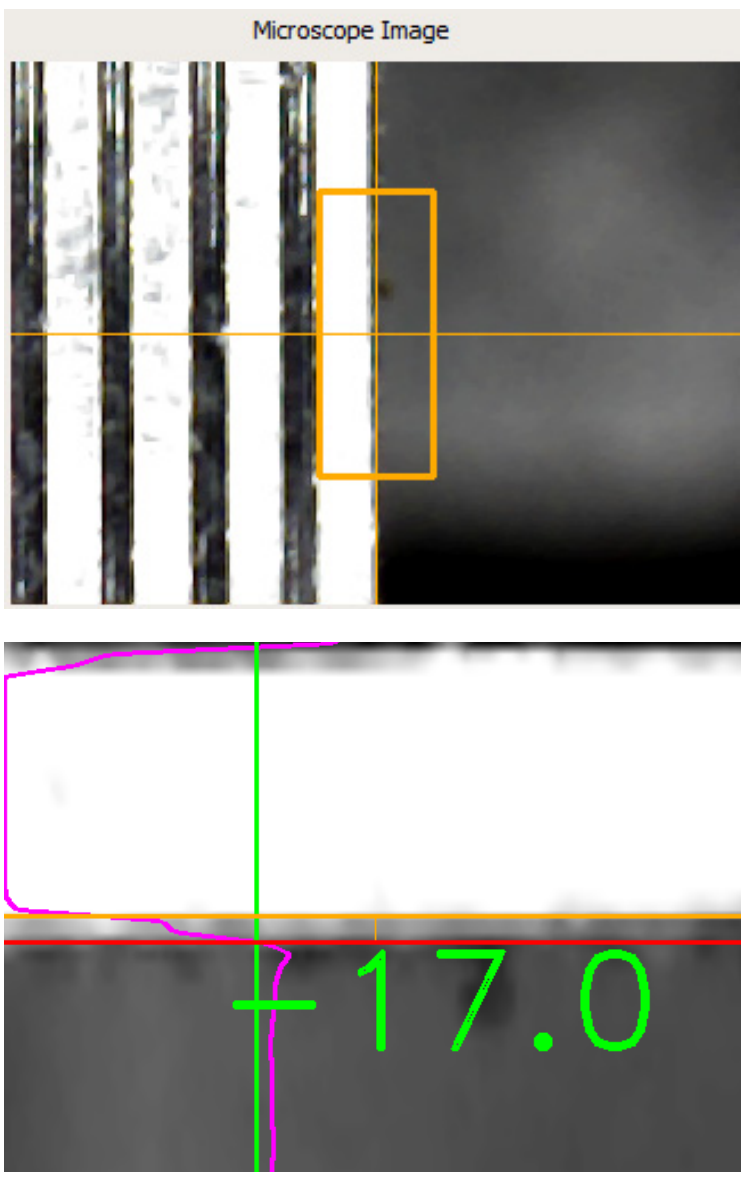

Fig. 12. Line edge images.

which illustrates the challenge with edge type rulers. The value displayed is in pixels, but was measured with laser interferometer as roughly $1.6 \mu \mathrm{m} /$ pixel. This gives a grey area height of about $26 \mu \mathrm{m}$.

This 'grey' area for this specific rule supports the systematic error found whilst comparing to the CMM results. Using the grey area as the main contributor for repeatability an estimated uncertainty of $38 \mu \mathrm{m}$ was calculated for calibration of rules with such edges with the absolute system (but will be dependent on individual rules). For none edge type rulers this can be significantly reduced to $17 \mu \mathrm{m}$ (for a $1 \mathrm{~m}$ rule).

This serves to illustrate that the line edge, as used for the measurement zero position has a significant contribution to the final measurement uncertainty. Segments measured from the rule edge, however do not need to conform to the OIML class accuracy (see [1] paragraph 4.2.4).

\subsection{Comparison method results}

The final test was with the comparator type system to determine the system measurement accuracy. This was done by comparing three rules which were calibrated with the absolute system. Furthermore it was assumed that the Rule for Re-verification Officers was the standard (see Fig. 1) and the other two rules the UUT. 


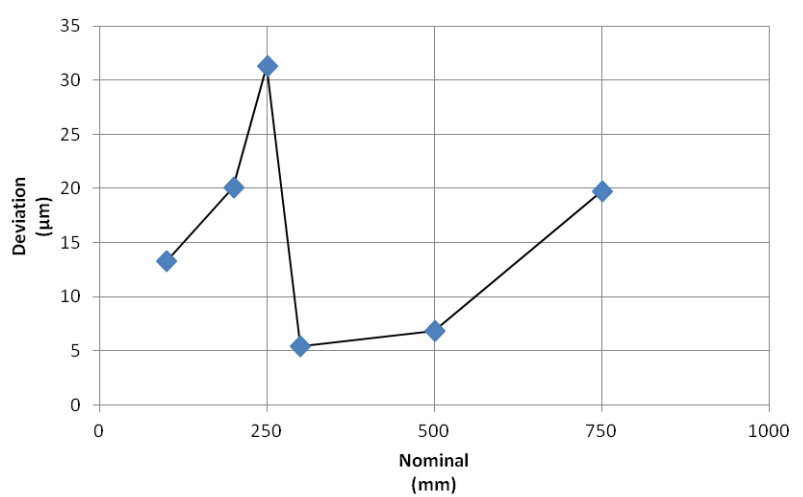

Fig. 13. Comparison test results for $900 \mathrm{~mm}$ edge type steel rule.

Figure 13 shows the results found for the $900 \mathrm{~mm}$ edge type steel rule. The maximum deviation was $31 \mu \mathrm{m}$ and the average $16 \mu \mathrm{m}$. The other rule had an average deviation of $18 \mu \mathrm{m}$ and a maximum of $26 \mu \mathrm{m}$.

All the deviations from the absolute calibration are positive, which could indicate a systematic error. Such errors can be corrected for by identifying the cause and then applying a correction factor, if possible, or modifying the measurement system as needed.

Ideally this deviation should be zero with a random distribution around the zero, based on the pixel noise and pixel to $\mu \mathrm{m}$ conversion factor.

\section{Preliminary comparison system uncertainty estimation}

Several assumptions have been mentioned previously, which if not perfectly valid, will affect the comparison system's uncertainty. The main uncertainty contributors which are discussed however are (1) the standard calibration uncertainty; (2) the UUT (and edge) line quality factor; (3) scale and image alignment; (4) temperature and (5) repeatability.

Repeatability here includes a per measurement repeatability due to 'noise', which includes stability of the scale to image ratio and so forth. This was determined by comparison of two calibrated rules, at different heights (distance between camera and scale) and settings. Furthermore, the camera calibration factor, which is unique for each rule calibration, along with a pixel noise estimate, can be used to determine a resolution and repeatability type uncertainty contributor. For example a $4 \mu \mathrm{m} /$ pixel value gives a resolution estimate of about $2 \mu \mathrm{m}$; with an estimate image noise of 2 pixels. It will depend on the measurement setup itself, but under approximately ideal conditions, the repeatability is estimated to be $(4+10) \mu \mathrm{m}$.

It is assumed that both the scales are at exactly the same temperature, the temperature measurement is perfect and the CTE is correct. This will not be the case. These effects are considered by the standard method, with an CTE uncertainty of $\pm 2 \mathrm{ppm}$ and a temperature measurement uncertainty of $0.1{ }^{\circ} \mathrm{C}$.

Scale alignment will include scale straightness, which will depend on the two individual scales. Such effects will also result in linear deviations. For now it is not considered and can be part of future studies.

Line and edge quality includes line straightness and any distortion which will result in lines not being precisely the same. This must be estimated for each rule individually. The measurement repeatability will include this effect and the half maximum range can be used for estimating this contributor, without adding an additional term.

Finally, calibration uncertainty of the standard used must be incorporated. If both rules however were measured with the same absolute system, it should be considered that the uncertainty of the comparison (as in Sect. 4.4) relative to the absolute system could be correlated.

\section{Conclusion}

The ALS-C (All Line Scale Comparator) can calibrate various rules and the results presented shows that the system can achieve $a \pm 50 \mu \mathrm{m}$ uncertainty for rule calibrations by comparison, which should be a useful and significant improvement relative to the current systems.

The system is developed in open source ensuring that continuous improvement is possible. Future work includes system validation and continuous investigation on reproducibility as well as any systematic errors.

After developing the comparison system, an absolute system with an integrated linear transducer and an automated positioning can be developed, which will again increase measurement accuracy and speed.

Finally, all of these improvements should improve African line scale and rule traceability and measurement capabilities.

Acknowledgements. The authors would like to thank Floris van der Walt for the CMM measurements of the rules and Faith Hungwe for technical review.

\section{References}

1. OIML, R35-1 Material measures of length for general use, OIML (2007)

2. Python Software Foundation (2013), http://www.python.org/

3. Open Source Computer Vision Library (2013), http://opencv.org/

4. Numerical Python (2013), http://www.numpy.org/

5. About PyQt (2013), http://wiki.python.org/moin/PyQt4

6. J. Matas, C. Galambos, J. Kittler, Robust detection of lines using progressive probabilistic Hough transform, Computer Vision and Image Understanding 78, 119-137 (2000) 